\title{
$A b$ initio simulation framework for Majorana transport in 2D materials: towards topological quantum computing
}

\author{
Y. Lee, T. Agarwal, and M. Luisier \\ Integrated Systems Laboratory, ETH Zurich, Zurich, Switzerland, email: youslee@iis.ee.ethz.ch
}

\begin{abstract}
We present an $a b$ initio modeling framework to simulate Majorana transport in 2D semiconducting materials, paving the way for topological qubits based on 2D nanoribbons. By combining density-functional-theory and quantum transport calculations, we show that the signature of Majorana bound states (MBSs) can be found in 2D material systems as zero-energy modes with peaks in the local density-of-states. The influence of spinorbit coupling and external magnetic fields on Majorana transport is studied for two relevant $2 \mathrm{D}$ materials, $\mathrm{WSe}_{2}$ and $\mathrm{PbI}_{2}$. To illustrate the capabilities of the proposed $a b$ initio platform, a device structure capable of hosting MBSs is created from a $\mathbf{P b I}_{2}$ nanoribbon and carefully investigated. These results are compared to InSb nanowires and used to provide design guidelines for 2D topological qubits.
\end{abstract}

\section{Introduction}

Quantum computer promises an exponentially augmented computing power to solve specific problems that are almost impossible to tackle using classical machines based on the von Neumann architecture [1]. Because of the large overhead caused by error correction, it is not yet clear if a sufficiently large number of qubits can be integrated with each other and if quantum supremacy can be reached in practical applications [2]. With this regard, topological qubits appear more robust than their superconducting and spin counterparts, owing to the encoding of the information in their topology [3].

To realize a topological qubit, Majorana Fermions, which are their own anti-particles, are needed [4]. They have been predicted to exist in solid-state systems such as 1D nanowires (NW) covered by a $p$-wave superconductor [5]. Last decade experiments have mostly focused on demonstrating the presence of Majorana bound states (MBS) in semiconductor-superconductor (SM-SC) interfaces, either with InSb or InAs nanowires covered by $\mathrm{NbTiN}$ or Al superconductors [6], [7], [8] or by using $\mathrm{Fe}$ atomic chains on a $\mathrm{Pb}$ superconducting surface [9]. The presence of MBSs in SM-SC systems follows the so-called "Majorana recipe", which summarizes requirements for the spinorbit coupling (SOC) and chemical potential of the semiconductor, superconducting gap, and Zeeman potential energy induced by external magnetic fields. Although the $g$-factor of InSb NWs make them particularly appealing as MBS host, fluctuations in their surfaces can lead to elusive signatures of Majorana particles.

2D semiconducting monolayers stacked on top of a superconductor, as illustrated in Fig. 1(a), offer an attractive alternative to NWs. Their ultra-narrow thickness allows to reduce the negative influence of structure variations and lead to stronger proximity effects with the adjacent superconductor, while still providing the Majorana conditions [10]. From a device perspective, tuning the chemical potential of the SM-SC interface can be more easily done with a $2 \mathrm{D}$ semiconductor than a covered nanowire. The operating principle of 2-D SM-SC configurations consists of leveraging the edge bands of nanoribbons (NR) made of 2-D monolayers with a (significantly) higher SOC than III-V NWs [11], e.g. WSe $\mathrm{W}_{2}$. A better detection of MBS, even with disorder, is then expected [10].

Atoms located at the edge of 2D NRs can induce edge states within the bandgap of the semiconductor. The latter can be used as MBSs, depending on the SOC strength. Designing such a system requires a high accuracy that only an $a b$ initio simulation environment can provide. In this work, we therefore present a comprehensive modeling framework to shed light on Majorana transport in 2D materials. It combines density-functional-theory (DFT) and quantum-transport calculations. The origin of Majorana Fermions is first explained in $\mathrm{WSe}_{2}$ and $\mathrm{PbI}_{2}$ monolayers that have been selected as testbeds due to their large SOC. Finite-size effects coming from the finite dimensions of the 2D NRs are then discussed by varying the device length within a fabrication-compatible range.

\section{Approach}

The most important ingredients of the Majorana recipe [12], [13], according to Kitaev's model [5], are listed in Fig. 1(b) for $2 \mathrm{D}$ materials. Here, a $\mathrm{PbI}_{2} \mathrm{NR}$ is chosen as example because: (i) it possesses an edge band well separated from the bulk bands and (ii) it shows a strong SOC in the edge band. Figure 1(b) compares a trivial state with Zeeman energy $V_{Z M}=0 \mathrm{meV}$ (no magnetic field) to a non-trivial topological one called Majorana zero-energy obtained at a "sweet spot" $\left(V_{Z M}=0.9895 \mathrm{meV}\right)$. It can be seen that this magnetic field can close the superconducting gap at the Majorana condition (MC), while increasing its magnitude reopens it.

Our $a b$ initio framework for Majorana quasi-particles is summarized in Fig. 2. First, the electronic structure of 2D NRs is computed with the VASP DFT package [15] within the generalized gradient approximation (GGA) of Perdew, Burke, and Ernzerhof (PBE) [16]. Tight-binding-like Hamiltonians are then created by projecting the plane-wave basis onto a set of maximally localized Wannier functions. Thereafter, the Tightbinding matrix elements of the $p$ and $d$ orbitals are grouped to form a basis for the $\vec{L} \cdot \vec{s}$ SOC operator [17]. The corresponding SOC parameters are chosen in such a way that the DFT bandstructure with SOC is accurately reproduced. The superconducting proximity effects are included by applying the 
Bogoliubov-de Gennes (BdG) transformation to the Wannier Hamiltonian, i.e. by changing the fermionic operators $\left(c^{\dagger}=\right.$ $\gamma_{1}-i \gamma_{2}$ and $\left.c=\gamma_{1}+i \gamma_{2}\right)$ to Majorana quasi-particle ones $\left(\gamma_{1}=\right.$ $\left(c^{\dagger}+c\right) / 2$ and $\left.\gamma_{2}=i\left(c^{\dagger}-c\right) / 2\right)$. Finally, the external magnetic field $B$ is accounted for along the transport direction through Zeeman's field, $V_{Z M}=g \mu_{B} B / 2$, and Pauli's spin matrices. The local density-of-states and transmission function of the finite systems are calculated with the Non-equilibrium Green's Function (NEGF) method with the produced BdG Hamiltonian.

\section{Results}

Using the proposed scheme, we study the Majorana condition in monolayer $\mathrm{PbI}_{2}$ and $\mathrm{WSe}_{2}$ NRs. The edge band of both materials exhibit significantly different electronic properties. Figure 3 (a) shows that the effective mass of the $\mathrm{WSe}_{2}\left(0.7 \mathrm{~m}_{0}\right)$ is smaller than that of $\mathrm{PbI}_{2}\left(1.8 \mathrm{~m}_{0}\right)$, while the SOC strength in $\mathrm{PbI}_{2}\left(\alpha_{S O}=0.88 \mathrm{eV} \AA, \mathrm{E}_{S O}=92.5 \mathrm{meV}\right)$ is considerably larger than in $\mathrm{WSe}_{2}\left(\alpha_{S O}=0.47 \mathrm{eV} \AA, \mathrm{E}_{S O}=10 \mathrm{meV}\right)$. Figure 3 (a) further indicates that a semiconductor with a larger $\alpha_{S O}$ has a smaller number of modes near zero energy, making the detection of Majorana Zero Modes (MZMs) more evident. Moreover, it is shown that the strength of the superconducting gap induced by the proximity effect $(\Delta)$ also affects the near-zero bands (highlighted in yellow): a smaller $\Delta$ reduces the density of nearzero energy modes (NZMs).

Next, Figs. 3 b) and (c) plot the topological phase diagrams of $\mathrm{PbI}_{2}$ and $\mathrm{WSe}_{2}$ monolayer NRs, respectively. It can be seen that the considered systems can be brought to a topological phase (presence of a MZM) from a trivial phase by either tuning the chemical potential of the $2 \mathrm{D}$ semiconductor or by varying the Zeeman potential. Such topological phase diagrams provide the much needed guidance to create a topological phase and subsequently a topological qubit.

To confirm the existence of MZMs, we can calculate the local-density-of-states (LDOS) of the 2-D semiconductors with our simulation framework. This quantity can be correlated to tunneling spectroscopy measurements [8]. Figures 4(a) and (b) show the energy-resolved LDOS as a function of the Zeeman potential for $\mathrm{PbI}_{2}$ and $\mathrm{WSe}_{2}$. We observe that the energy gap gradually closes in the materials as the magnetic field increases before opening again after the Majorana condition is reached, i.e. at $V_{Z M}=0.9895 \mathrm{meV}\left(\mathrm{PbI}_{2}\right)$ and $0.734 \mathrm{meV}\left(\mathrm{WSe}_{2}\right)$. The MZMs correspond to the zero-energy mode peaks in Fig. 4(c). It should be noted that the length of the semiconductor NR has been so far assumed infinite and its electrostatic potential has been kept constant and homogeneous, thus preventing the investigation of possible finite-size effects.

To go one step further, we now consider the device structure of Fig. 5(a), where the influence of the finite size effect is introduced through the electrostatic potential depicted in Fig. 5. a) and the generation of NRs made of up to 140,000 atoms. By varying the height of the tunneling barrier, we can assess the impact of the 2D NR length on the properties of the MZMs. Figure 5(b) shows the energy-resolved transmission function with respect to the barrier height $\Phi_{T B}$ and the NR length $L$, highlighting the MZM and NZMs. Near-zero modes corresponding to new bound states appear within the superconducting gap, which is set to $\Delta=0.1 \mathrm{meV}$ here. As the device length increases, the number of bound states within the gap grows and the NZMs move closer to zero energy. In Fig. 5 ( c), the energy difference between NZMs is extracted as a function of the device length. From this data, an important parameter called critical length ( $\left.L_{\text {crit }}\right)$ can be inferred. It provides a condition for the device length $L$, which should satisfy $L>>L_{\text {crit }}$ to observe a strong MZM signature.

Finite-size effects can be further identified in Figs. 6(a) and (b) where the LDOS is shown for two device lengths $L=1.34$ and $5.53 \mu \mathrm{m}$, one below and one above $L_{\text {crit }}=3.3 \mu \mathrm{m}$. We can clearly see the presence of localized NZMs at both extremities of the device, as expected for MBSs [18]. Moreover, the NZMs move closer to the zero energy when the device length increases, as is also visible in Fig. 5. The MZM and NZMs, plotted for different lengths, provide another evidence of the localization of these states at the device extremities. Finally, our results clearly indicate that the device length should be larger than $L_{\text {crit }}$ to obtain a stronger MZM localization.

\section{Conclusion}

We have developed an $a b$ initio simulation framework to explore the feasibility of topological qubits in nanoribbons made of 2D monolayer semiconductors. Transport calculations with up to 140,000 atoms have been performed in 2D NRs to observe MZM and topological phases. The Table in Fig. 6.c) gives an overview of the physical settings to create a MZM in $\mathrm{WSe}_{2}$ and $\mathrm{PbI}_{2} \mathrm{NRs}$ and compare them to the same parameters for InSb nanowires. Our modeling environment opens new avenues for the design of robust topological qubits with long decoherence times.

\section{Acknowledgment}

This work was supported the MARVEL National Centre of Competence in Research of the Swiss National Science Foundation (SNSF), by SNSF under Grant No. 175479 (ABIME), and by a grant from the Swiss National Supercomputing Centre (CSCS) under Project s876.

\section{References}

[1] R. P. Feynman, Int. J. Theor. Phys. 21, 467-488 (1982).

[2] F. Arute et al., Nature 574, 505-510 (2019).

[3] R. Raussendorf et al., 2007 New J. Phys. 9199

[4] E. Majorana, Il Nuovo Cimento (1924-1942) 14, 171 (2008).

[5] A. Y. Kitaev, Phys. Uspekhi 44, 131-136 (2001).

[6] V. Mourik et al., Science 336, 1003-1007 (2012).

[7] M. T. Deng et al. Science 354, 1557-1562 (2016).

[8] H. Zhang et al. Nature 556, 74-79 (2018).

[9] S. Nadj-Perge et al., Science 346, 602-607 (2014).

[10] R.-L. Chu et al., Phys. Rev. B 89, 155317 (2014).

[11] A. Pal et al., Proc. of IEDM 2019, 24.2.1-24.2.4 (2019).

[12] R. M. Lutchyn et al., Phys. Rev. Lett. 105, 077001 (2010)

[13] Y. Oreg et al., Phys. Rev. Lett. 105, 177002 (2010)

[14] Y. Nagamune et al., Phys. Rev. B 43, 12401 (1991)

[15] G. Kresse and J. Furthmüller, Phys. Rev. B 54, 11169 (1996).

[16] J. P. Perdew et al., Phys. Rev. Lett. 77, 3865 (1997).

[17] S. Konschuh et al., Phys. Rev. B 82, 245412 (2010).

[18] R. M. Lutchyn et al. Nat. Rev. Materials, 3(5), 52-68 (2018).

[19] T. Karzig et al. Phys. Rev. B 95, 235305 (2017). 

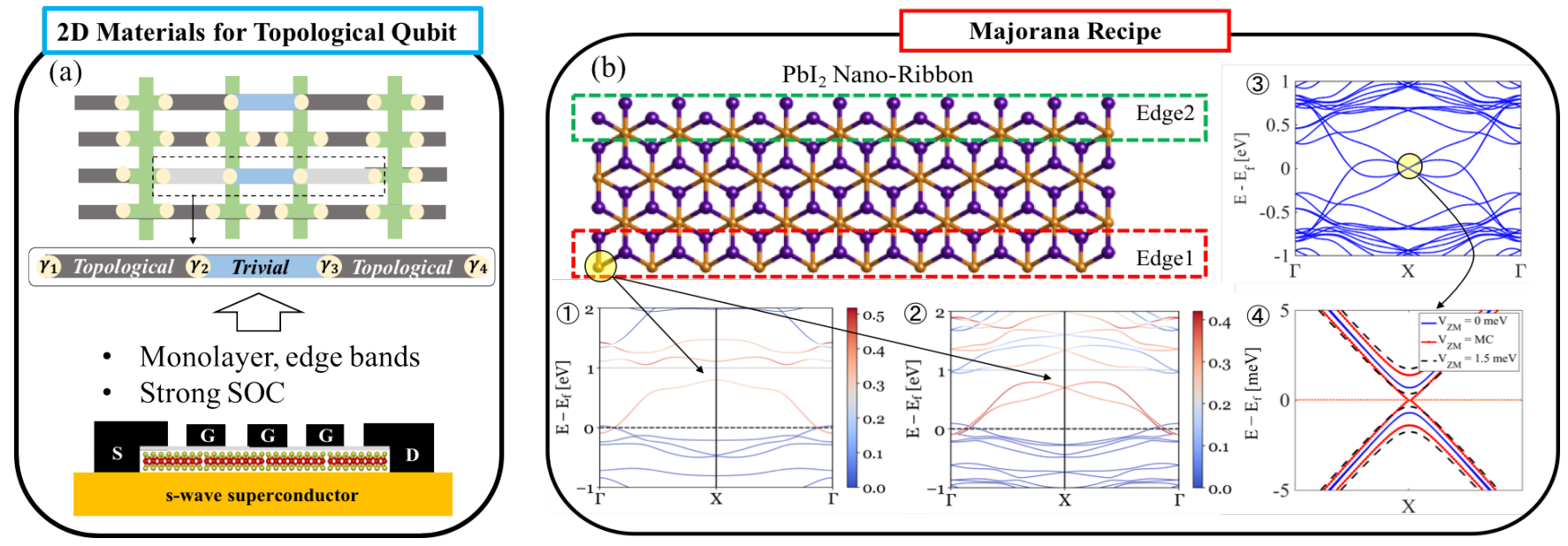

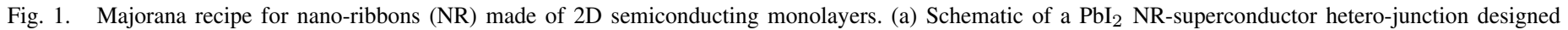

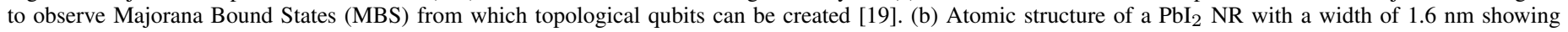
the contribution of the edge atoms to the electronic bandstructure computed with VASP without (1) and with spin-orbit coupling (2). Sub-plot (3) reports the particle-hole BdG bandstructure accounting for proximity effects caused by an $s$-wave superconductor stacked on top of the 2D NR. Sub-plot (4) displays the band modulation through the Zeeman potential induced by an external magnetic field.

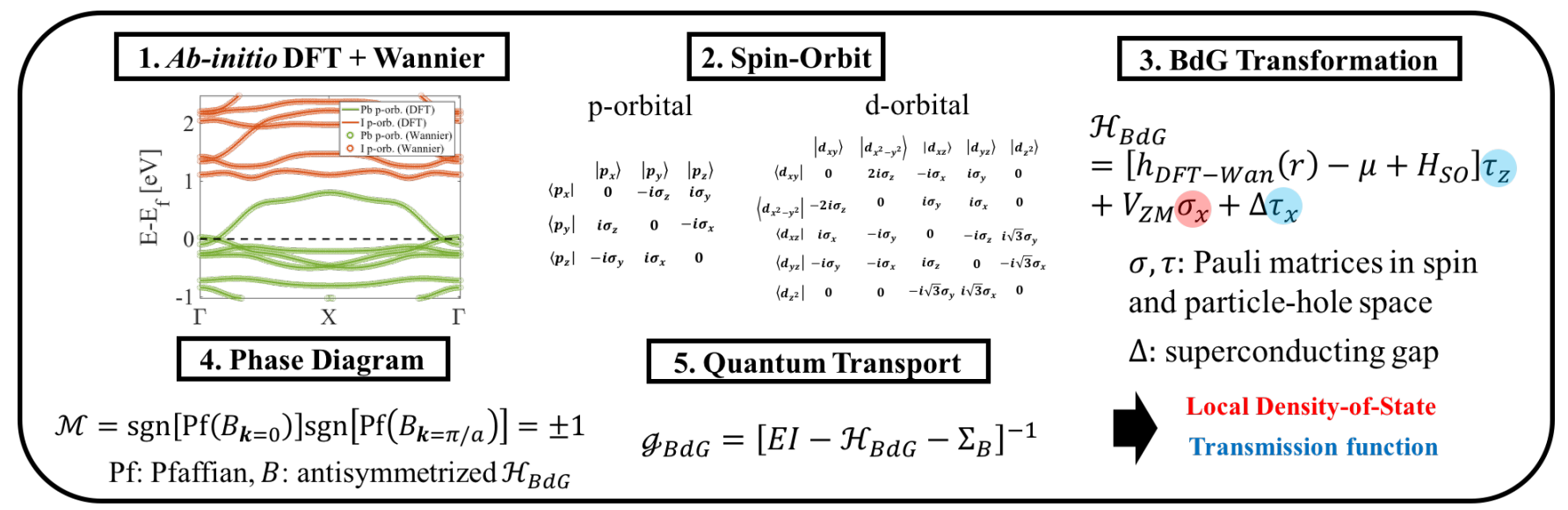

Fig. 2. Summary of the proposed ab initio simulation framework. (1) The electronic bandstructure obtained from density-function-theory (DFT) is first projected to a Wannier function basis (here: $\mathrm{PbI}_{2}$ ). (2) Inclusion of $\mathrm{SOC}$ in the Wannier Hamiltonian to reproduce the DFT bandstructure with SOC. (3) BdG transformation of the Wannier Hamiltonian to capture the influence of the superconductor stacked on top of the 2D material. (4) Determination of the topological phase with the help of Pfaffian invariants. (5) NEGF-based quantum transport calculations using the BdG Hamiltonian. 

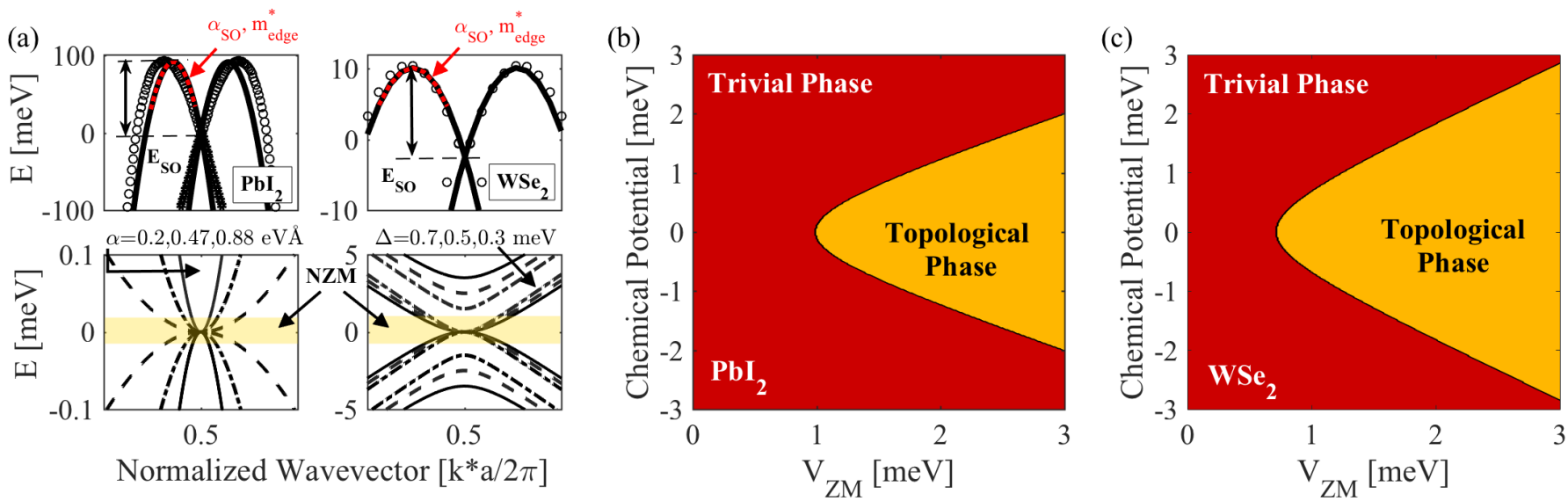

Fig. 3. (a,top) Extraction of the effective mass of the edge band $\left(\mathrm{m}_{\text {edge }}^{*}\right)$, spin-orbit energy $\left(\mathrm{E}_{S O}\right)$, and spin-orbit coupling strength $\left(\alpha_{S O}\right)$ from the DFT bandstructure with SOC (symbols). The solid lines represent the fit obtained with a 1D Majorana model [13]. (a,bottom) Effect of $\alpha_{S O}$ and the superconducting gap $(\Delta)$ on topological states at the Majorana condition. Here, NZM refer to near-zero energy modes, which are the modes in the highlighted region. (b) Topological phase diagram ( $x$-axis: Zeeman potential energy, $y$-axis: chemical potential of the material) of $\mathrm{PbI}_{2}$. (c) Same as (b), but for WSe 2 .
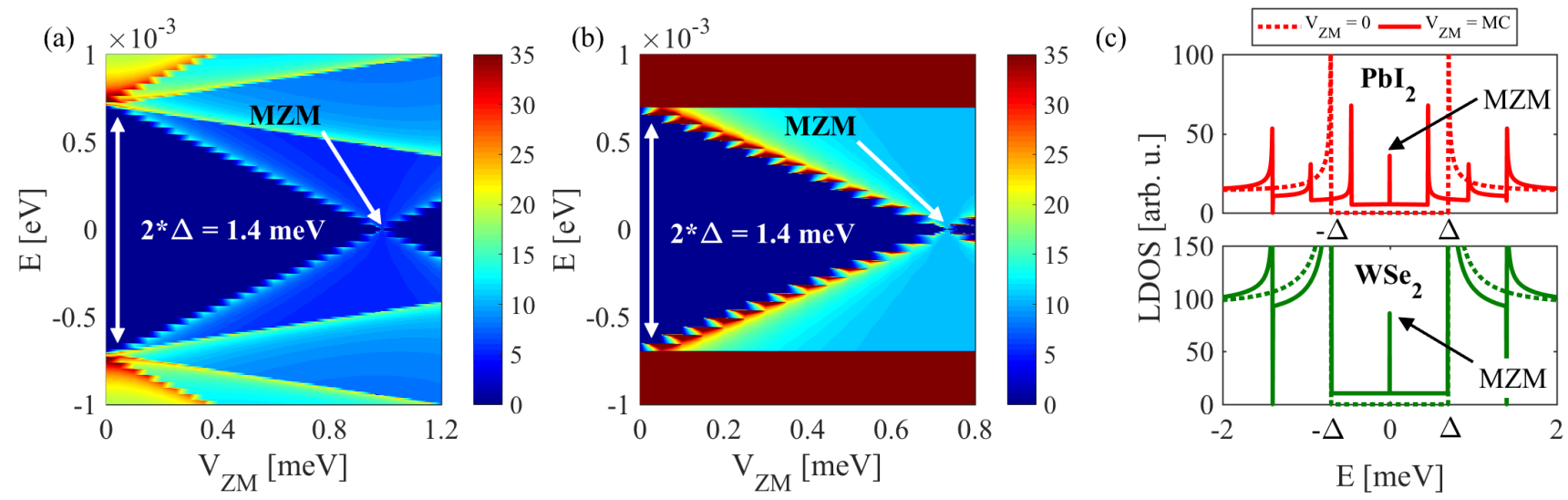

Fig. 4. (a) Energy-resolved local-density-of-states (LDOS) of $\mathrm{PbI}_{2}$ as a function of the Zeeman potential energy $V_{Z M}$. (b) Same as (a), but for WSe 2 . (c) Same as (a) and (b), but for $V_{Z M}=0 \mathrm{meV}$ (dahsed line) and for the $V_{Z M}$ providing the Majorana condition (MC, solid lines). A superconducting gap $\Delta=0.7 \mathrm{meV}$ is assumed. The MZM peaks are clearly visible when $V_{Z M}$ is tuned to the Majorana condition (MC), where $V_{Z M}=g \mu_{B} B / 2, g$ : landé $g$-factor, $\mu_{B}$ : magnetic moment, B: external magnetic field. 


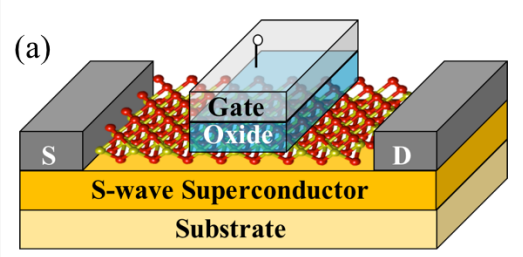

Potential Profile

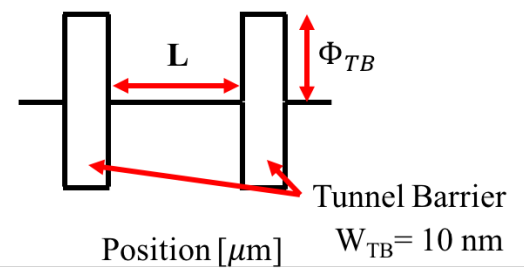

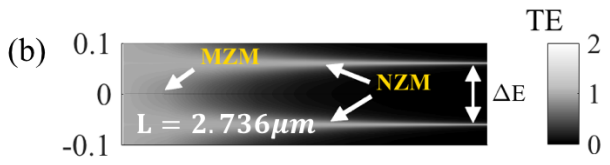

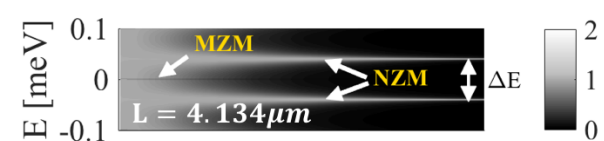

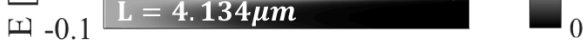

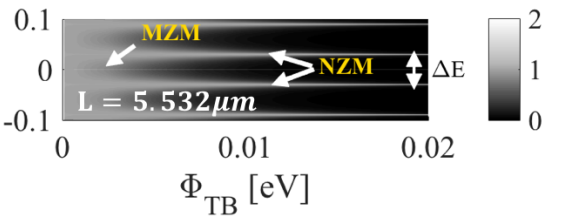

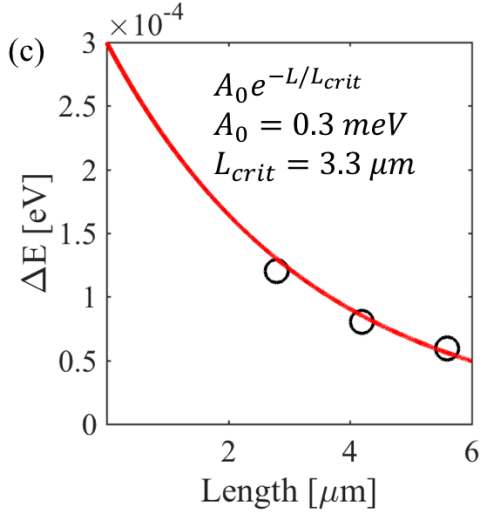

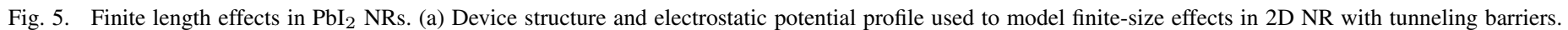

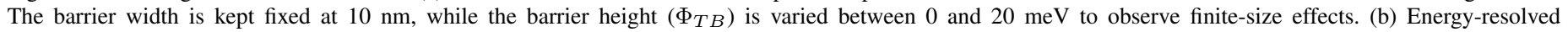

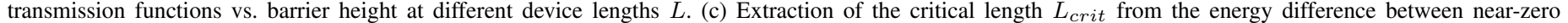
energy modes $(\Delta \mathrm{E})$ as a function of the device length $L$.

(a)

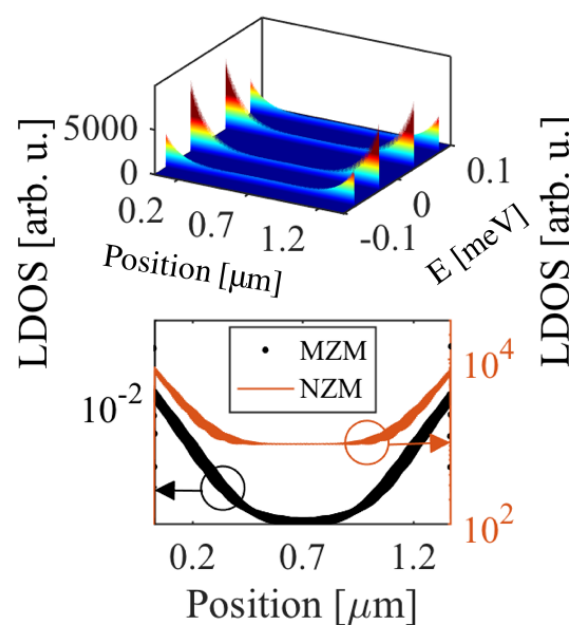

(b)

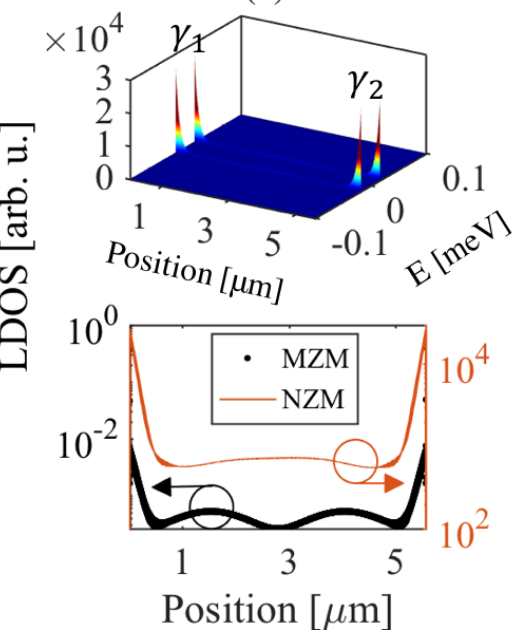

\begin{tabular}{|c|c|c|c|}
\hline (c) & $\mathrm{PbI}_{2}$ & $\mathrm{WSe}_{2}$ & $\mathrm{InSb}[18]$ \\
\hline Structure & $\mathrm{NR}$ & $\mathrm{NR}$ & $\mathrm{NW}$ \\
$\mathrm{MZM}$ transport & Edge & Edge & Bulk \\
$\mathrm{E}_{S O}[\mathrm{meV}]$ & 92.5 & 10 & $0.05-1$ \\
$\alpha_{S O}[\mathrm{eV} \AA]$ & 0.88 & 0.47 & $0.2-1$ \\
$\lambda_{S O}[\mathrm{~nm}]$ & 0.48 & 2.3 & $230-50$ \\
$L_{\text {crit }}[\mu \mathrm{m}]$ & $3-4$ & - & $1-2$ \\
$\lambda_{M F P}[\mathrm{~nm}]$ & - & - & $200-300$ \\
Width $[\mathrm{nm}]$ & 1.6 & 2 & $80-100$ \\
Thickness [nm] & 0.68 & 0.65 & $80-100$ \\
Proximity effect & High & High & Low \\
$m^{*}\left[m_{0}\right]$ & 1.8 & 0.7 & 0.014 \\
$g$-factor & - & - & $40-50$ \\
\hline
\end{tabular}

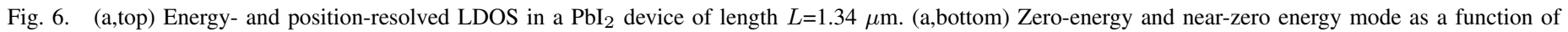

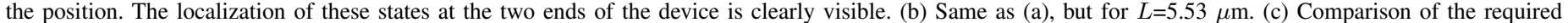
physical settings in $\mathrm{WSe}_{2}$ and $\mathrm{PbI}_{2}$ nanoribbons and in $\mathrm{InSb}$ nanowires to build a topological qubit based on a Majorana bound state. 